\title{
DESIGN OF BEAM-EXTRACTION SEPTUM MAGNET FOR THE SNS*
}

\author{
N. Tsoupas, Y. Y. Lee, J. Rank, and J. Tuozzolo BNL, Upton, NY 11934, USA
}

\section{Abstract}

The beam-extraction process from the SNS accumulator ring [1,2] requires a Lambertson septum magnet. In this paper we discuss the geometrical and magnetic field requirements of the magnet and present results obtained from two and three dimensional magnetic field calculations that shows the field quality in the regions of interest of the septum magnet.

\section{INTRODUCTION}

The beam extraction from the SNS accumulator ring is a single turn two step process. In the first step, the single bunch containing $2 \times 10^{14}$ protons at an energy of $1 \mathrm{GeV}$ is "kicked" vertically by a set of 14 kickers into the high field region of a septum magnet, and in the second step, the bunch is deflected left $16.8^{0}$ on the outside of the ring by the extraction septum magnet into the RTBT beamtransfer-line to the SNS target. The septum magnet is of Lambertson type and the optimization of the design was based on $1 \mathrm{GeV}$ protons and in this paper we report on:

- the Electromagnetic design (2D and 3D calculations)

- Mechanical design

\section{ELECTROMAGNETIC DEGIGN}

\subsection{Two Dimensional Design}

The SNS fast-extraction kickers "kicks" the beam vertically and displace it from the horizontal level of the ring to the entrance of the septum magnet. In order to maximize the vertical displacement of the extracted beam at the entrance of the septum magnet, the septum magnet was placed as far as possible from the kickers. The location of the septum magnet in conjunction with the angle of bend and beam size, defines a set of parameters for the septum magnet which appear in Table 1.

Table 1 Parameters of the septum magnet

\begin{tabular}{|c|c|c|c|c|c|}
\hline $\begin{array}{c}\mathrm{L}_{\text {eff }} \\
{[\mathrm{m}]}\end{array}$ & $\begin{array}{c}\mathrm{B} \\
{[\mathrm{T}]}\end{array}$ & $\theta_{\text {bend }}$ & $\begin{array}{c}\text { gap }_{\text {extr }} \\
{[\mathrm{cm}]}\end{array}$ & $\begin{array}{c}\text { aper }_{\text {circ }} \\
{[\mathrm{cm}]}\end{array}$ & $\begin{array}{c}\text { Thick }_{\text {sep }} \\
{[\mathrm{cm}]}\end{array}$ \\
\hline 2.44 & 0.68 & $\begin{array}{l}16.8 \\
{ }^{\circ}\end{array}$ & 16.9 & 17.5 & 1.0 \\
\hline
\end{tabular}

The aperture of the magnet for the circulating beam is $17.5 \mathrm{~cm}$ with acceptance of $480 \pi \mathrm{mm} . \mathrm{mrad}$. The magnetgap in the beam-extraction region is $16.9 \mathrm{~cm}$ with acceptance of $460 \pi \mathrm{mm} . \mathrm{mrad}$. This acceptance is determined by the vertical beam-size and the requirement that the beam must be extracted without losses even when one of the 14 kickers has not fired. The septum thickness is $1.0 \mathrm{~cm}$

The aim of the 2D calculations is two-fold; first to determine the minimum cross section of the magnet which will prevent saturation of the iron and second to optimize the field uniformity.

Figure 1 shows a cross section at the entrance of the magnet normal to the beam direction. The circular hole in the magnet iron is the aperture of the circulating beam. The contour plot of the field $\mathrm{B}_{\text {mod }}$ over the hole shows that the circulating beam experiences fields within the range of 1 to $\sim 10$ Gauss.

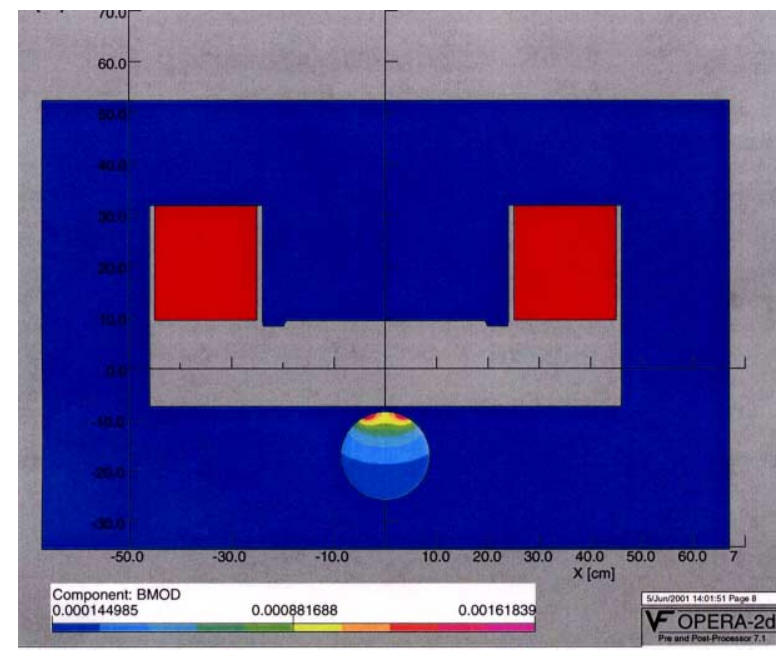

Figure 1: Cross-section of the septum magnet.

The strength of the normal $\left(b_{n}\right)$ and skew $\left(a_{n}\right)$ multipoles determine the field uniformity inside the magnet. The relative contributions of the magnetic multipoles to the dipole field as a function of radius $r$ (in meters), is: $\left(b_{n} / b_{0}\right) r^{n}$ for the normal multipoles and $\left(a_{n} / b_{0}\right) r^{n}$ for the skew. The most significant ratios $\left(b_{n} / b_{0}\right)$ and $\left(\mathrm{a}_{\mathrm{n}} / \mathrm{b}_{0}\right)$ appear in Table 2. The field expansion coincides with the central trajectory of the extracted beam.

Table 2. Contributions of the normal and skew multipoles.

\begin{tabular}{|c|c|c|c|}
\hline \multicolumn{2}{|c|}{$\left(\mathrm{b}_{\mathrm{n}} / \mathrm{b}_{0}\right) \mathrm{r}^{\mathrm{n}}$ Normal } & \multicolumn{2}{|c|}{$\left(\mathrm{a}_{\mathrm{n}} / \mathrm{b}_{0}\right) \mathrm{r}^{\mathrm{n}}$ Skew } \\
\hline $\begin{array}{c}\text { Sext. } \mathrm{n}=2 \\
{\left[\mathrm{~m}^{-2}\right]}\end{array}$ & $\begin{array}{c}\text { Dec. } \mathrm{n}=4 \\
{\left[\mathrm{~m}^{-4}\right]}\end{array}$ & $\begin{array}{c}\text { Quad } \mathrm{n}=1 \\
{\left[\mathrm{~m}^{-1}\right]}\end{array}$ & $\begin{array}{c}\text { Octup. } \mathrm{n}=3 \\
{\left[\mathrm{~m}^{-3}\right]}\end{array}$ \\
\hline-0.03 & 1.12 & 0.08 & 4.3 \\
\hline
\end{tabular}

\footnotetext{
* Work performed under the auspices of the DOE
} 


\subsection{Three Dimensional Design}

The 2D calculations on the septum magnet determines: a) the cross section of the pole and of the "return-iron" and b) the field uniformity of the main field region and the field strength in the circulating field region well inside the magnet. The $3 \mathrm{D}$ calculations determine the magnetic field at the entrance and exit of the septum magnet for both the extraction field region and the circulating beam region and provide information on the effect of the "magnetic shielding" that is appropriate to reduce the field strength in the circulating field region. In order to reduce the design effort and the computer time of the $3 \mathrm{D}$ calculations we studied the entrance and the exit of the magnet in two separate models, discussed in the following subsections.

\subsubsection{D study of the entrance of the magnet}

An isometric view of the entrance of the septum magnet is shown in figure 2 .

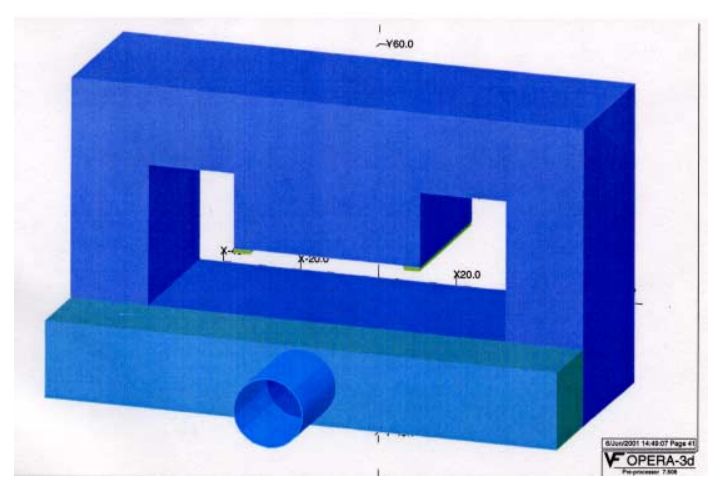

Figure 2: Isometric view of the magnet's entrance.

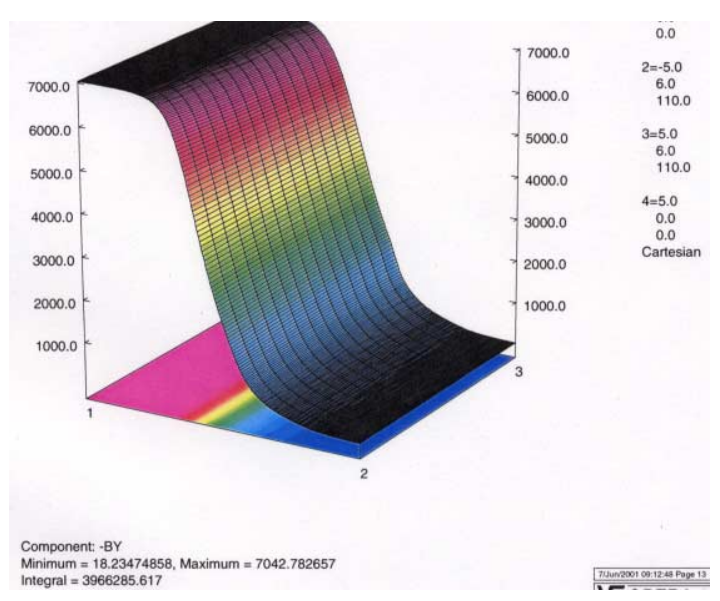

Figure 3: The field By at the entrance of the magnet

The coil of the magnet has been omitted to allow the viewing of the shims on the sides of the top pole face. The dimensions of these shims determine the field uniformity well inside the magnet. The "extension_shield" and the "pipe-shield", showing are made of the same magnetic material as the main body of the magnet, and reduce the field (see fig. 4) at the region of the circulating beam.

The field is plotted over a rectangle with coordinates shown in the upper right corner of the plot. The plane of the rectangle is defined by the direction of the central ray of the extracted beam (z-axis) and the direction parallel to the pole face of the magnet(x-axis).

The calculated value of the (EFB) Effective Field Boundary is $10 \mathrm{~cm}$ outside the pole face of the magnet.

The "extension_shield" and the "pipe-shield", (both shown in fig. 2) have a significant effect on the magnetic field at the region of the circulating beam. This effect is shown in figure 4 which plot the field $B_{\text {mod }}$ along the direction of the circulating beam at the entrance of the septum magnet, with and without magnetic "shielding".

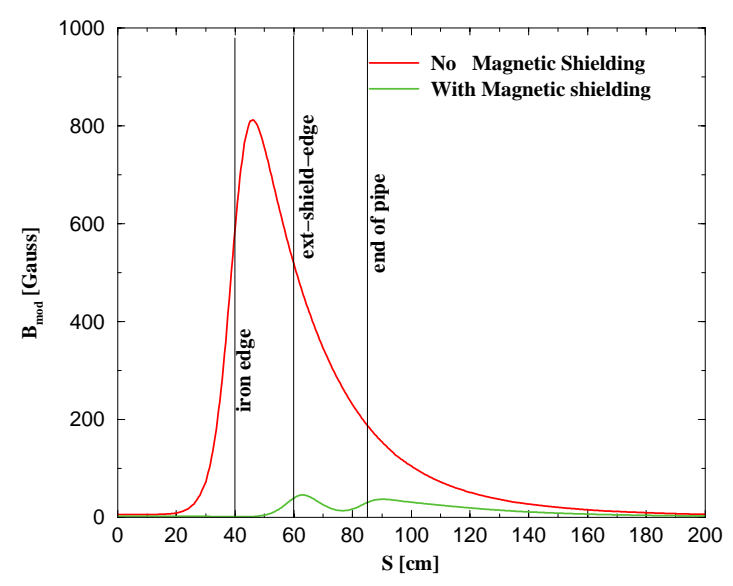

Figure 4: The $\mathrm{B}_{\text {mod }}$ vs distance along the circulating beam. The origin of the $\mathrm{x}$-axis is $40 \mathrm{~cm}$ inside the magnet, as measured from the external edge of the iron. The length of the "extension-shield" and "pipe_shield" is 20 and $25 \mathrm{~cm}$.

The magnetic multipoles of the stray fields along the circulating beam at the entrance of the magnet (with "shields"), have been calculated and have been added to the corresponding magnetic multipoles at the exit and to those within the magnet. The integrated magnetic multipoles which are introduced by the septum magnet on the circulating beam, appear in Table 3.

Table 3. The most significant integrated multipoles along the circulating beam direction.

\begin{tabular}{|c|c|c|c|}
\hline \multicolumn{2}{|c|}{ Normal } & \multicolumn{2}{c|}{ Skew } \\
\hline $\begin{array}{c}\int b_{0} d z \\
\text { [Gauss.cm] }\end{array}$ & $\begin{array}{c}\int \mathrm{b}_{1} \mathrm{dz} \\
{[\mathrm{Gauss}]}\end{array}$ & $\begin{array}{c}\int \mathrm{a} \text { dz } \\
{[\text { Gauss.cm] }}\end{array}$ & $\begin{array}{c}\int \mathrm{a}_{1} \mathrm{dz} \\
{[\text { Gauss }]}\end{array}$ \\
\hline 2200 & -100 & 4700 & 200 \\
\hline
\end{tabular}

\subsubsection{D study of the exit of the septum magnet}

The geometry of the septum magnet at the exit differs from that at the entrance. An isometric view of the septum magnet at the exit is shown in figure 5. In order to minimize the field interference between the septum magnet and a ring quadrupole(located at a distance of $\sim 45$ 
$\mathrm{cm}$ from the exit of the magnet), we allow only an "extension shield" (see figure 5) at the exit of the magnet, for stray field reduction. The reduction of the stray field along the circulating beam directions, caused by the "extension-shield" at the exit of the magnet, is shown in figure 6 .

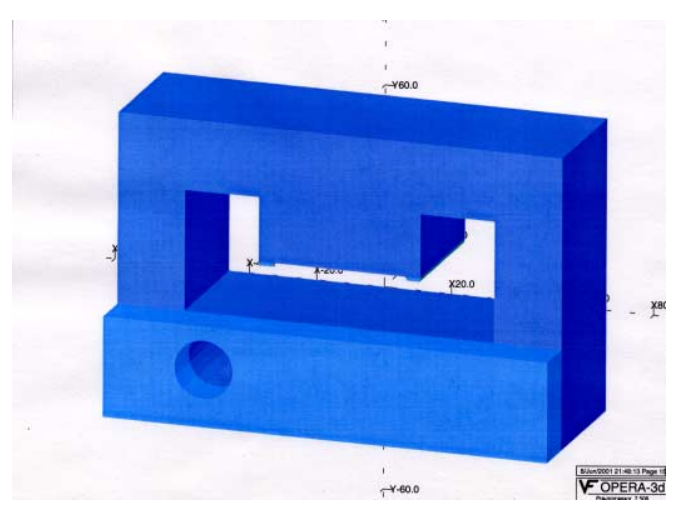

Figure 5: Isometric view of the septum magnet at the exit

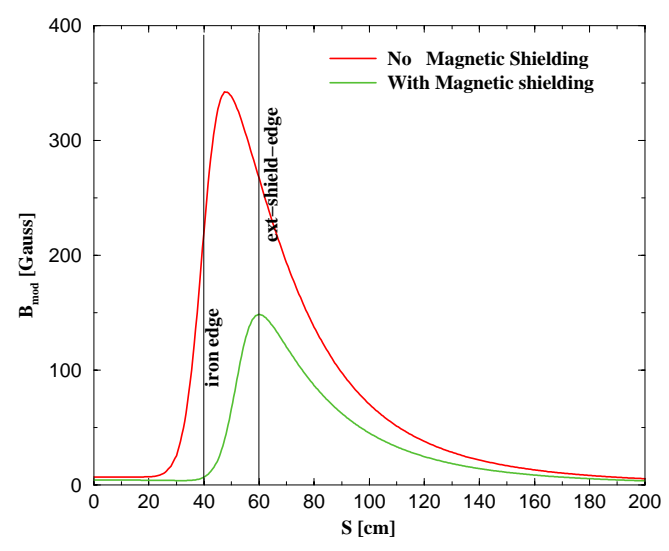

Figure 6: The $\mathrm{B}_{\text {mod }}$ vs distance along the circulating beam

\section{BASIS OF THE MECHANICAL DESIGN}

The $2 \mathrm{D}$ and $3 \mathrm{D}$ calculations were performed to determine the geometry of the septum magnet which will provide adequate field uniformity in the beam extraction region and minimize the stray fields that will affect the circulating beam.

There are however additional design constraints that stem from the following requirements: a) The bend angle of the magnet $\left(16.8^{0}\right)$ b) the direction of the extracted beam with respect to the direction of the circulating beam $(13.95 \mathrm{mrad})$ and $\mathrm{c})$ the beam size at the entrance and exit of the septum magnet. These requirements are summarized in figure 7 which is the projection of the magnet iron on a vertical plane containing the circulating beam.

In order to fulfill these requirements and also minimize the thickness of the septum iron, the following constraints should be imposed on the mechanical design: a) the bottom of the circulating beam should be parallel to the surface of the beam pipe ("S1 surface" fig. 7) for the circulating beam $b$ ) the top surface of the extracted beam should parallel to the surface the magnet pole ("S2 surf fig. 7). c) the surface ("S3 surface" fig. 7) should be parallel to the surface $\mathrm{S} 1$ and at an adequate distance to allow for the growth of the beam size.

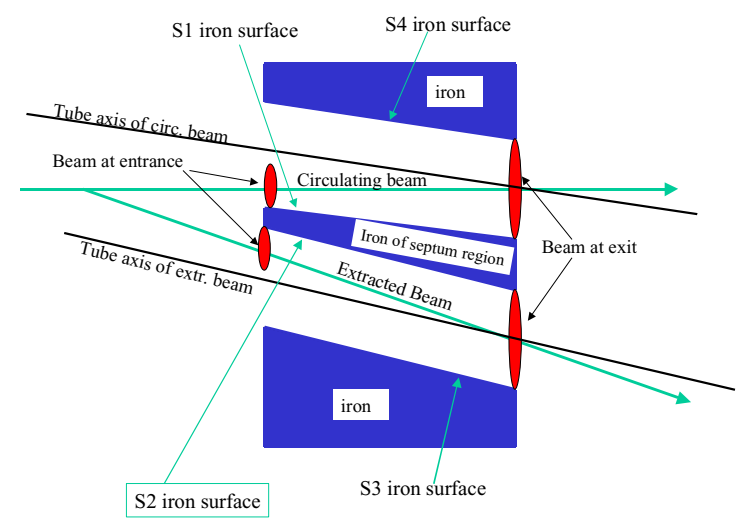

Figure 7: Schematic diagram of the septum cross section along the beam.

The surface labeled "S4 surface" in fig. 7 can be made to coincide with the top surface of the circulating beam. Such a beam pipe however is rather expensive to make. We therefore use a cylindrical beam pipe.

\section{CONCLUSIONS}

A preliminary design for the SNS extraction septum magnet shows that magnet correctors may be necessary to compensate for the field multipoles introduced by the magnet on the circulating beam at the entrance and exit of the magnet. Alternative "shielding" approaches [4] are under consideration.

\section{REFERENCES}

[1] N. Tsoupas et. "Beam Extraction from the SNS Ring and Design of theExtraction Kickers." Seventh EPAC Vienna, Austria

[2] N. Tsoupas et. al. "Chromatic Correction and Optical Compensation in the SNS Accumulator Ring Using

Sextupoles" Seventh EPAC Vienna, Austria

[3]Vector-Fields-Inc

[4] N. Tsoupas et. al. "Design and B-Field Measurements of A Lambertson Injection Magnet for the RHIC

Machine" PAC 1995 page 1352 